\title{
Kinetics of gas-phase hydrogen/deuterium exchange and gas-phase structure of protonated phenylalanine, proline, tyrosine and tryptophan
}

\author{
Marko Rožman, Saša Kazazić, Leo Klasinc and Dunja Srzić
}

Site-specific rate constants for the gas-phase hydrogen/deuterium (H/D) exchange of four, three, five and five hydrogen atoms in protonated phenylalanine (Phe), proline (Pro), tyrosine (Tyr) and tryptophan (Trp), respectively, were determined from matrix-assisted laser desorption/ionization Fourier transform ion cyclotron resonance mass spectrometry (MALDI-FTICRMS) experiments with $\mathrm{D}_{2} \mathrm{O}, \mathrm{D}_{2} \mathrm{~S}$, and $\mathrm{CH}_{3} \mathrm{OD}$ as deuterating agents. No $\mathrm{H} / \mathrm{D}$ exchange was observed with $\mathrm{D}_{2} \mathrm{~S}$. For exchange with both $\mathrm{CD}_{3} \mathrm{OD}$ and $\mathrm{D}_{2} \mathrm{O}$, which is about ten times slower in the latter, results indicate for all compounds protonation of the $\alpha$-amino group in agreement with theoretical results. Also, with both reagents, all compounds exchange at the $\mathrm{COOH}$ site more than ten times faster than at the protonation site, with $\mathrm{OH}$ and $\mathrm{NH}$ sites of Tyr and Trp, respectively, exchanging slowest. The observation of H/D exchange despite the high differences in proton affinities between the amino acids and deuterating agent exceeding $200 \mathrm{~kJ}$ mol $^{-1}$ is in agreement with lowering of the barrier for proton transfer through hydrogen bonding proposed by Lebrilla and coworkers.

Over the past two decades, mass spectrometry has become an important tool for analyses of biomolecules. In particular, the introduction of matrix-assisted laser desorption/ionization $^{1,2}$ (MALDI) and electrospray ionization ${ }^{3,4}$ (ESI) has made study of biomolecules by mass spectrometry (MS) widely used. Besides characterization and identification of these molecules, some techniques, e.g. hydrogen/deuterium exchange, ${ }^{5}$ also enable structural investigation. The H/D exchange between the neutral biomolecule and the deuterating agent may take place in the condensed phase or between a charged biomolecule and the neutral deuterating agent in the gas phase within the mass spectrometer. The subsequently recorded mass spectrum serves to monitor the site and extent of the exchange reaction. The structural information obtained in this way may differ if the solution and gas-phase structures differ. Thus, amino acids which are zwitterions in solution tend to have free molecule structures in the gas phase. First indication for such gas-phase structures came from photoelectron (PE) spectra of aliphatic amino acids, ${ }^{6,7}$ which showed that their electronic structures corresponded with the normal molecules. This conclusion was confirmed also by correlation of PE spectra for the pairs (amino acid/amino acid methyl ester) vs. (carboxylic acid/carboxylic acid methyl ester), ${ }^{8}$ for which correlation would not exist if the amino acids were zwitterions; this is impossible for their esters. However, such proof could not be presented for those amino acids which decompose on heating. Thus, gas-phase $\mathrm{H} / \mathrm{D}$ exchange studies of amino acids remain of particular interest because they also enable the determination of the structures of positively and negatively charged molecular ions as well as of protonated (or else cationized) forms of amino acids. Such studies may also provide relative exchange rates for different sites, location of the charge and dependence on the deuterating agent. For all these answers it is necessary to confine the charged species for a long enough time in the mass spectrometer to perform and monitor the $\mathrm{H} / \mathrm{D}$ exchange experiment in the gas phase.

Development of ion-trapping techniques such as Fourier transform ion cyclotron resonance (FTICR) and the quadrupole ion trap are such methods of choice. ${ }^{5}$ Both methods are capable of trapping ions over long periods of time while they are undergoing collisions with a deuteration reagent. This capability is an important feature that allows observation of the relatively slow $\mathrm{H} / \mathrm{D}$ exchange processes.

The generally accepted simplified gas-phase H/D exchange mechanism consists of three steps: $: 9,10$ the initial formation of a loose hydrogen-bonded complex between a protonated substrate and a deuteration reagent, $\mathrm{H} / \mathrm{D}$ exchange within the complex and dissociation of the complex to yield either the original or the exchange species. For exchange to be observed, the energy made available by complex formation must be sufficient to overcome the barrier for internal proton transfer. This barrier depends mainly on the proton affinity difference between the two unprotonated species $\left(\triangle \mathrm{PA}=\mathrm{PA}_{\text {substrate }}-\mathrm{PA}_{\text {reagent }}\right)$. In the earlier studies ${ }^{11}$ of the $H / D$ exchange it was assumed that if $\Delta \mathrm{PA}>84 \mathrm{~kJ} \mathrm{~mol}^{-1}$, there will be no exchange reaction. However, it was shown $\mathrm{n}^{5,12,13}$ that the $\mathrm{H} / \mathrm{D}$ exchange reaction will proceed even when $\Delta \mathrm{PA}>210 \mathrm{~kJ} \mathrm{~mol}^{-1}$. Occurrence of 
exchange was explained ${ }^{14}$ by the formation of multiple hydrogen bonds within the exchange complex because multiple hydrogen bonds are expected to lower the barrier for the internal proton transfer.

There are two ways of treating the kinetics of gas-phase H/D exchange reactions..$^{5,12,15}$ The first ${ }^{5,12,14}$ is to treat the system as one of successive exchanges with apparent rate constants $\left(\mathrm{k}_{\mathrm{a}}, \mathrm{k}_{\mathrm{b}}, \ldots\right)$. For example, a system with two exchangeable hydrogen atoms:

$$
\mathrm{D}_{0} \stackrel{\mathrm{k}_{\mathrm{a}}}{\longrightarrow} \mathrm{D}_{1} \stackrel{\mathrm{k}_{\mathrm{b}}}{\longrightarrow} \mathrm{D}_{2}
$$

where $D_{0}, D_{1}$ and $D_{2}$ denote molecules containing zero, one and two deuterium atoms in place of hydrogen atoms. The other way $^{5,15,16}$ to treat the system as $n$ independent exchangeable sites, each with its site-specific rate constants $\left(k_{1}, k_{2}, \ldots\right)$, yields for the same example, with the two exchangable hydrogen atoms:

$$
\begin{aligned}
& \mathrm{HH} \stackrel{\mathrm{k}_{1}}{\longrightarrow} \mathrm{HD} \stackrel{\mathrm{k}_{2}}{\longrightarrow} \mathrm{DD} \\
& \mathrm{HH} \stackrel{\mathrm{k}_{2}}{\longrightarrow} \mathrm{DH} \stackrel{\mathrm{k}_{1}}{\longrightarrow} \mathrm{DD}
\end{aligned}
$$

where $D_{1}$ species observed in a system is the sum of two species HD and DH. It has been shown $n^{5,15,16}$ that site-specific rate constants give better insights into the structure of the analyte molecule and reaction mechanism because this treatment actually starts by presuming them. So it is possible to assign and confirm site-specific gas-phase H/D exchange rate constants by regression using the experimental data. Site-specific treatment of H/D exchange reaction on protonated glycine ${ }^{5,15}$ indicated one fast exchanging site (carboxyl group) and three equivalent slow exchanging sites (amino group). The results provided evidence for a structure of protonated glycine in which the proton is localized on the amino group.

In this work, by using MALDI-FTICRMS, the H/D exchange reactions of protonated amino acids: phenylalanine (Phe), proline (Pro), tyrosine (Tyr) and tryptophan (Trp), with $\mathrm{D}_{2} \mathrm{O}, \mathrm{D}_{2} \mathrm{~S}$, and $\mathrm{CH}_{3} \mathrm{OD}$ as deuteration reagents are investigated. Our aim is to confirm that formation of hydrogen bonds between substrate and deuteration reagent plays a significant role in lowering the barrier for internal proton transfer within the reaction complex. Using the site-specific treatment of the gas-phase H/D exchange reactions on the studied amino acids the most favorable protonation site of each amino acid is determined and compared with theoretical calculation results. ${ }^{17}$

\section{EXPERIMENTAL}

The H/D exchange experiments were performed in a $3 \mathrm{~T}$ Fourier transform ion cyclotron resonance (FTICR) mass spectrometer with a Nicolet 1280 data station (Extrel FTMS 2001; Madison, USA) equipped with a nitrogen laser (VSL 337 NSD; LSI Laser Science, Newton, USA) emitting at $\lambda=337 \mathrm{~nm}$. All amino acids were from Fluka (Buchs, Switzerland). The deuteration reagents $\mathrm{D}_{2} \mathrm{O}(99.8 \%)$ and $\mathrm{D}_{2} \mathrm{~S}(97 \%)$ were from Aldrich (Milwaukee, USA). $\mathrm{CD}_{3} \mathrm{OD}(99.8 \%)$ was from Cambridge Isotope Laboratories (Andover, USA).

Before each experiment deuterating gas was introduced for several hours directly into the ICR cell for stabilization.

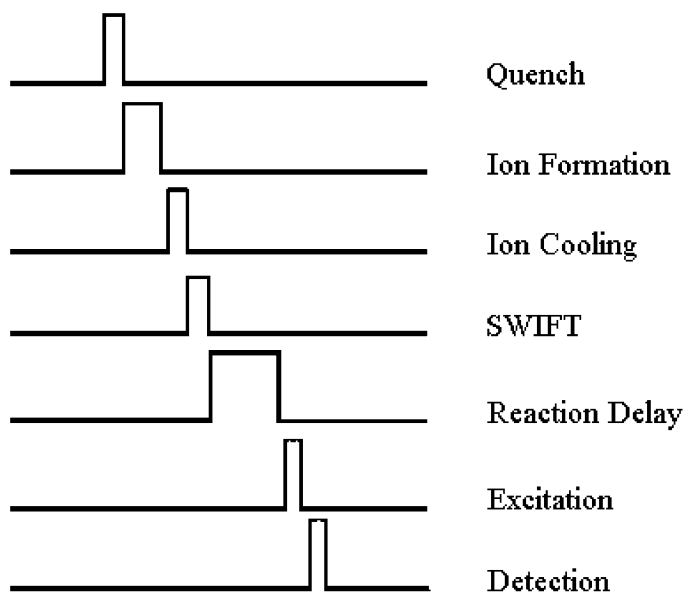

Figure 1. FTICR mass spectrometry experimental event sequence for each reaction period of the gas-phase $H / D$ exchange experiment.

Reagent gas pressure used in the exchange experiments ranged from $2.7 \times 10^{-5} \mathrm{~Pa}$ to $6.7 \times 10^{-6} \mathrm{~Pa}$ and was measured with a Bayard-Alpert-type ionization gauge using parameterized response factors for calibration. ${ }^{18}$ All experiments were performed at an ambient temperature of $300 \mathrm{~K}$.

Ions were formed by MALDI from samples prepared with a standard dried-droplet procedure using 2,5-dihydroxybenzoic acid (DHB) as matrix. Strongest ion signals were obtained with a sample-to-matrix ratio of $\sim 1: 100$.

The experimental event sequence is shown in Fig. 1. Ions trapped in the ICR cell were allowed to cool for $1 \mathrm{~s}$. Stored waveform inverse Fourier transform (SWIFT) excitation was used to isolate monoisotopic ions of the protonated amino acid, $[\mathrm{M}+\mathrm{H}]^{+}$. These were then allowed to react for a certain period of time with a deuterating gas. To monitor the exchange kinetics after each reaction period all ions were detected by their mass spectrum, thus yielding a series of spectral data for the reaction investigated. A typical exchange experiment lasted up to $150 \mathrm{~s}$ with a dozen of spectra taken. The duration of the total length of the experiment is limited by the instrument performance.

Data obtained from mass spectra consist of relative intensities of the mass peaks for $D_{0}, D_{1}, \ldots, D_{n}$ (where $D_{n}$ represents an ion species containing a total of $n$ deuterium atoms) measured at a number of time points that correspond to different time delay for the exchange reaction. The kinetic system is treated as $n$ independent exchangeable hydrogen atoms each following a simple pseudo first-rate law. With this approach we determined site-specific rate constants of studied protonated amino acids. An algorithm similar to that recently proposed by $\mathrm{He}$ and Marshall ${ }^{15}$ was used to write an interactive program for calculation of site-specific rate constants in Mathematica 4.0 (Wolfram Research, Champaign, USA).

\section{RESULTS AND DISCUSSION}

The proton affinities ${ }^{19}$ and strengths of hydrogen bonds for the three deuteration reagents: $\mathrm{CD}_{3} \mathrm{OD}, \mathrm{D}_{2} \mathrm{~S}$ and $\mathrm{D}_{2} \mathrm{O}$, which were allowed to react in the ICR cell with the protonated amino acids Phe, Pro, Tyr and Trp, are given in Table 1. The 
Table 1. Proton affinity and strength of the hydrogen bonds of the deuteration reagents used

\begin{tabular}{lccc}
\hline & $\mathrm{CD}_{3} \mathrm{OD}$ & $\mathrm{D}_{2} \mathrm{~S}$ & $\mathrm{D}_{2} \mathrm{O}$ \\
\hline $\mathrm{PA}\left(\mathrm{kJ} \mathrm{mol}^{-1}\right)$ & 754.7 & 703.3 & 690.7 \\
Hydrogen bond & Strong & Weak & Strong \\
\hline
\end{tabular}

Table 2. Proton affinity differences $(\triangle P A)$ between unprotonated amino acid (AA) and deuteration reagent (in $\mathrm{kJ} \mathrm{mol}^{-1}$ )

\begin{tabular}{lcccc}
\hline Amino acid & Phe & Pro & Tyr & Trp \\
\hline PA (AA) & 923.0 & 920.5 & 924.7 & 987.4 \\
PA(AA)-PA(CD $\left.C_{3} \mathrm{OD}\right)$ & 168.3 & 165.8 & 170.0 & 194.2 \\
PA(AA)-PA(D $\left.\mathrm{D}_{2} \mathrm{O}\right)$ & 232.3 & 229.8 & 234.0 & 258.2 \\
PA(AA)-PA(D $\left.\mathrm{D}_{2} \mathrm{~S}\right)$ & 219.7 & 217.2 & 221.4 & 245.6 \\
\hline
\end{tabular}

proton affinity ${ }^{19}$ differences $(\triangle \mathrm{PA})$ between amino acids and deuteration reagents examined in this work are given in Table 2.

As mentioned above it was assumed ${ }^{11}$ that no H/D exchange reaction will be possible if $\Delta \mathrm{PA}>84 \mathrm{~kJ} \mathrm{~mol}^{-1}$. However, H/D exchange reactions were shown $n^{5,12,13}$ to proceed even when $\Delta \mathrm{PA}>210 \mathrm{~kJ} \mathrm{~mol}^{-1}$, which was attributed to a lowering of the barrier for the proton transfer within the reaction complex by formation of multiple hydrogen bonds. Such a hydrogen-bonded complex of a simple protonated amino acid and alcohol, as proposed by Gard et al., ${ }^{14}$ is shown in Fig. 2.

Table 2 shows that all values of $\Delta \mathrm{PA}$ are higher than $84 \mathrm{~kJ} \mathrm{~mol}^{-1}$, and that some of them are even higher than $210 \mathrm{~kJ} \mathrm{~mol}^{-1}$. Gas-phase H/D exchange is observed in all amino acids with $\mathrm{CD}_{3} \mathrm{OD}$ and $\mathrm{D}_{2} \mathrm{O}$, but none is observed with $\mathrm{D}_{2} \mathrm{~S}$. This result indicates that formation of the hydrogen bonds between substrate and deuteration reagent may indeed play a significant role in lowering the barrier for internal proton transfer within the H/D exchange complex. Although $\mathrm{D}_{2} \mathrm{~S}$ does not have the highest proton affinity difference it forms the weakest hydrogen bonds. These weak hydrogen bonds within the reaction complex amino acid$\mathrm{D}_{2} \mathrm{~S}$ do not sufficiently lower the barrier for the internal proton transfer and there is no H/D exchange reaction with $\mathrm{D}_{2} \mathrm{~S}$ in the gas phase.

The protonated amino acids studied in this work were produced from amino acids Phe, Pro, Tyr and Trp by MALDI, which typically generates protonated gas-phase ions.<smiles>[R]O[I][NH2+]C([R2])C(=O)O[2H]</smiles>

Figure 2. Hydrogen-bonded complex between a protonated amino acid and deuteration reagent.<smiles>O=C(O)C1CCCN1</smiles><smiles>NC(Cc1ccccc1)C(=O)O</smiles>

$\operatorname{Pr}$

Phe

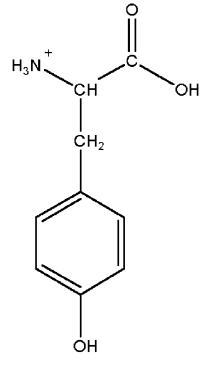

Tyr<smiles>NC(Cc1c[nH]c2ccccc12)C(=O)O</smiles>

$\operatorname{Trp}$
Figure 3. Most favorable protonation site of the studied amino acids. ${ }^{14}$

Because there are several possible protonation sites in the amino acid structures, the most favorable protonated forms were calculated ${ }^{17}$ and were all found to correspond to the amino group protonation (see Fig. 3). With the site-specific treatment of the gas-phase $H / D$ exchange rates it is possible to confirm the most favorable protonation site of each amino acid (see Fig. 4).

Tables 3 and 4 summarize site-specific gas-phase H/D exchange rate constants obtained for the protonated amino acids studied. Repetitive H/D exchange experiments indicate a relative standard deviation of $20 \%$ for the reported sitespecific rate constants.

The site-specific treatment of $\mathrm{H} / \mathrm{D}$ exchange of protonated Phe with $\mathrm{CD}_{3} \mathrm{OD}$ and $\mathrm{D}_{2} \mathrm{O}$ indicates one fast exchanging site and three equivalent slow exchanging sites. The fast exchanging site can be attributed to the carboxylic group and the three equivalent slow exchanging sites to the

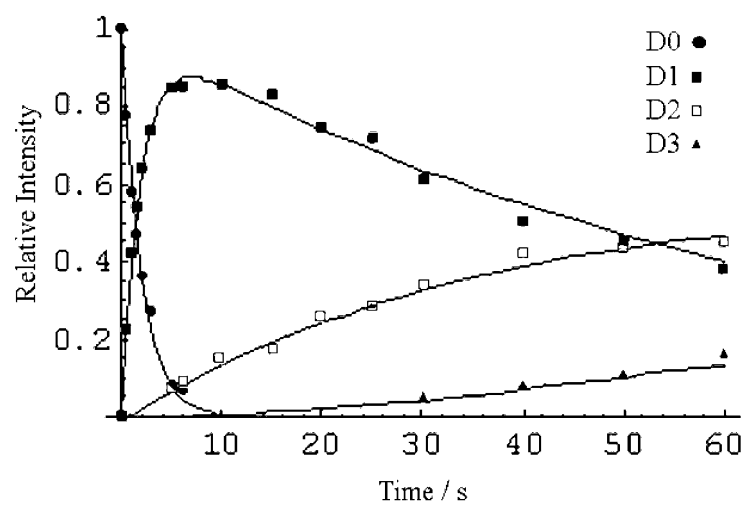

Figure 4. Time dependence of the experimental ion intensities and corresponding theoretical fit obtained with the sitespecific treatment of the $\mathrm{H} / \mathrm{D}$ exchange in proline with $\mathrm{D}_{2} \mathrm{O}$. D0, D1, D2, and D3 represent the relative abundances of protonated proline in which $0,1,2$, or 3 hydrogen atoms have been replaced by deuterium. 
Table 3. Site-specific gas-phase H/D exchange rate constants $\left(k_{i} \pm 20 \%\right)$ for protonated amino acids studied $\left(\times 10^{-12} \mathrm{~cm}^{3} \mathrm{~s}^{-1}\right.$ molecule $\left.{ }^{-1}\right)$ with $\mathrm{CD}_{3} \mathrm{OD}$

\begin{tabular}{lcccc}
\hline & Phe & Pro & Tyr & Trp \\
\hline Pressure (Pa) & $3.33 \times 10^{-5}$ & $6.65 \times 10^{-6}$ & $4.00 \times 10^{-5}$ & $5.33 \times 10^{-6}$ \\
$\mathrm{k}_{1}$ & 86.92 & 630.5 & 885.77 & 275.91 \\
$\mathrm{k}_{2}$ & 9.31 & 37.79 & 7.05 & 7.13 \\
$\mathrm{k}_{3}$ & 9.31 & 37.79 & 7.05 & 7.13 \\
$\mathrm{k}_{4}$ & 9.31 & - & 7.05 & 7.13 \\
$\mathrm{k}_{5}$ & - & - & 1.53 & 1.26 \\
\hline
\end{tabular}

Table 4. Site-specific gas-phase $H / D$ exchange rate constants $\left(k_{i} \pm 20 \%\right)$ for protonated amino acids studied $\left(\times 10^{-12} \mathrm{~cm}^{3} \mathrm{~s}^{-1}\right.$ molecule $\left.{ }^{-1}\right)$ with $\mathrm{D}_{2} \mathrm{O}$

\begin{tabular}{lcccc}
\hline & Phe & Pro & Tyr & Trp \\
\hline Pressure (Pa) & $3.47 \times 10^{-5}$ & $4.67 \times 10^{-5}$ & $3.33 \times 10^{-5}$ & $4.67 \times 10^{-5}$ \\
$\mathrm{k}_{1}$ & 11.01 & 36.59 & 10.32 & 14.72 \\
$\mathrm{k}_{2}$ & 0.46 & 5.59 & 0.92 & 0.69 \\
$\mathrm{k}_{3}$ & 0.46 & 5.59 & 0.92 & 0.69 \\
$\mathrm{k}_{4}$ & 0.46 & - & 0.92 & 0.69 \\
$\mathrm{k}_{5}$ & - & - & 0.39 & 0.1 \\
\hline
\end{tabular}

protonated amino group, in agreement with the theoretical result. ${ }^{17}$

For protonated Pro one fast exchanging site and two equivalent slow sites are observed. The latter indicate the amino group as site of protonation, which is also in agreement with the calculated structure. ${ }^{17}$

Site-specific treatment of the H/D exchange reaction of protonated Tyr indicates one fast exchanging site, i.e. the carboxylic group, three equivalent slow exchanging sites, i.e. the protonated amino group, and one slow exchanging site, i.e. the phenol $\mathrm{OH}$ group site. The result confirms the calculated structure ${ }^{17}$ of protonated Tyr with the proton localized at the amino group.

Trp has the highest proton affinity difference $(\triangle \mathrm{PA})$ of all the amino acids studied here, but it also undergoes $H / D$ exchange of five atoms with $\mathrm{CD}_{3} \mathrm{OD}$ and $\mathrm{D}_{2} \mathrm{O}$. It has several possible protonation sites and calculation indicates that the proton is localized on the $\alpha$-amino group. ${ }^{17}$ The site-specific rate constants for protonated Trp indicate the presence of three identical and two dissimilar hydrogen atoms, i.e. a fast exchanging carboxylic and the slowly exchanging one of the indole amino group, all in agreement with the calculation.

The $\mathrm{H} / \mathrm{D}$ exchange rate constants with $\mathrm{CD}_{3} \mathrm{OD}$ are roughly one order of magnitude larger than with $\mathrm{D}_{2} \mathrm{O}$. For both reagents the rate of $\mathrm{H} / \mathrm{D}$ exchange at the $\mathrm{COOH}$ site is $10-100$ times faster then for the $\mathrm{NH}_{3}^{+} / \mathrm{NH}_{2}^{+}$site. The $\mathrm{OH}$ site of Tyr and $\mathrm{NH}$ site of Trp have the lowest exchange rates. Also for both reagents the rate constants are highest for Pro, followed by Tyr and lowest for Phe. It is interesting to note, however, that all the $\mathrm{NH}_{3}^{+}$site exchange rates are basically the same but significantly 5-10 times slower than $\mathrm{NH}_{2}^{+}$exchange rates for the same deuteration agent.

\section{CONCLUSIONS}

Gas-phase H/D exchange in the protonated amino acids Phe, Pro, Trp and Tyr was found to occur with $\mathrm{CD}_{3} \mathrm{OD}, \mathrm{D}_{2} \mathrm{O}$ but not with $\mathrm{D}_{2} \mathrm{~S}$, although, due to large differences in proton affinities of the neutral amino acids and the deuterium donors, no exchange is predicted for all of them. The suggestion ${ }^{5,14}$ that formation of hydrogen bonds within the exchange complex enables the reaction agrees with the present result because $\mathrm{D}_{2} \mathrm{~S}$ forms the weakest hydrogen bond.

The obtained site-specific reaction rate constants confirm the gas-phase structure of the investigated protonated amino acids as protonation of their amino group. This finding is also in agreement with theoretical results. ${ }^{17}$ Although the major source of error in the numerical values of the sitespecific rate constants is about $20 \%$, arising mainly from the pressure measurement, the relationship between the rates of exchange for the sites in each experiment remains unaffected by the number of experimental runs.

\section{Acknowledgement}

Helpful discussions with Professor Biserka Kojić-Prodić are gratefully acknowledged.

\section{REFERENCES}

1. Tanaka K, Waki H, Ido Y, Akita S, Yoshida Y, Yoshida T. Rapid Commun. Mass Spectrom. 1988; 2: 151.

2. Hillenkamp F, Karas M, Beavis RC, Chait BT. Anal. Chem. 1991; 63: 1193A.

3. Yamashita M, Fenn JB. J. Phys. Chem. 1984; 88: 4671.

4. Fenn JB, Mann M, Meng CK, Wong SF, Whitehouse CM. Science 1989; 246: 64 .

5. Green MK, Lebrilla CB. Mass Spectrom. Rev. 1997; 16: 53.

6. Debies TP, Rabalais JW. J. Electron Spectrosc. 1974; 3: 315.

7. Klasinc L. J. Electron Spectrosc. 1976; 8: 161.

8. Klasinc L. Int. J. Quantum. Chem. 1978; QBS5: 373.

9. Lias SG. J. Phys. Chem. 1984; 88: 4401.

10. Green MK, Gard E, Bregar J, Lebrilla CB. J. Mass. Spectrom. 1995; 30: 1103.

11. Ausloos P, Lias SG. J. Am. Chem. Soc. 1981; 103: 3641.

12. Gard E, Willard D, Bregar J, Green MK, Lebrilla CB. Org. Mass Spectrom. 1993; 28: 1632.

13. Campbell S, Rodgers MT, Marzluff EM, Beauchamp JL. J. Am. Chem. Soc. 1995; 117: 12840.

14. Gard E, Green MK, Bregar J, Lebrilla CB. J. Am. Soc. Mass Spectrom. 1994; 5: 623.

15. He F, Marshall AG. J. Phys. Chem. A 2000; 104: 562.

16. He F, Marshall AG, Freitas MA. J. Phys. Chem. B 2001; 105: 2244.

17. Maksić ZB, Kovačević B. Chem. Phys. Lett. 1999; 307: 497.

18. Bartmess JE, Georgiadis RM. Vacuum 1983; 33: 149.

19. Hunter EP, Lias SG. J. Phys. Chem. Ref. Data 1998; 27: 413-656 (NIST Chemistry WebBook). 